\title{
Reply to the letter about inadequate depth of nasal tube
}

\author{
Ha Yeon Kim ${ }^{1}$. Jeongmin $\mathrm{Kim}^{2}$ \\ Received: 16 January 2019 / Accepted: 22 January 2019 / Published online: 9 March 2019 \\ (c) Japanese Society of Anesthesiologists 2019
}

To the editor:

Our anesthesiologist chose the tube sizes based on inner diameter 7.0 for male and 6.0 for female, and adjusted them considering patient's height and airway anatomy. The tube depth after intubation was assessed by combining various methods, including direct view of the tube cuff during intubation (at least $2 \mathrm{~cm}$ below the vocal cord), auscultation using a stethoscope, palpitation of the tube cuff at the patient's sternal notch, and analyses of airway pressure curves.

In fact, in some patients, the position of tube at the start of surgery might be already not proper, because we did not confirm the tube depth immediately after intubation. However, we had experienced that the nasal RAE tube occasionally became endobronchial intubation immediate after intubation and required the depth adjustment. There could be some patients with deep tube depth within carina $<2 \mathrm{~cm}$ which was not detected. Interestingly, there was no patient with a deep tube on postoperative chest radiography. Hence, we thought that the intraoperative factors would affect the shallow tube depth.

As the previous literature cited in the reference [1], the changes in tube fixation depth were poorly correlated with the changes in the tube distal tip, even if there is no noticeable distortion of the tube. The previous literature and our results means that even though the patient's head returned to the neutral position after surgery, the tube tip would not have returned to its original position. Therefore, we think that the depth of tube tip should be re-evaluated at the end of surgery because the reliability of tube fixation depth is poor.

\section{Compliance with ethical standards}

Conflict of interest The authors declare that they have no competing interests.

\section{Reference}

1. Wang ML, Schuster KM, Bhattacharya B, Maung AA, Kaplan LJ, Davis KA. Repositioning endotracheal tubes in the intensive care unit: depth changes poorly correlate with postrepositioning radiographic location. J Trauma Acute Care Surg. 2013;75:146-9.

Publisher's Note Springer Nature remains neutral with regard to jurisdictional claims in published maps and institutional affiliations.

This reply refers to the comment available online at https://doi. org/10.1007/s00540-019-02615-7.

Jeongmin Kim

Anesjeongmin@yuhs.ac

1 Department of Anesthesiology and Pain Medicine, Ajou University School of Medicine, 164 World cup- ro, Yeongtong-gu, Suwon 16499, Republic of Korea

2 Department of Anesthesiology and Pain Medicine, Anesthesia and Pain Research Institute, Yonsei University College of Medicine, 50 Yonsei-ro, Seodaemun-gu, Seoul 03722, Republic of Korea 